\title{
A Behavioural Assessment Unit improves outcomes for patients with complex psychosocial
} needs.

Professor George Braitberg MBioethics, MHIthServMt, Dip Epi Biostats, FACEM, FACMT

Associate Professor Marie Gerdtz RN, PhD

Susan Harding RN

Associate Professor Jonathan Knott MClinEd, PhD, FACEM

All authors were involved in the design of the study and preparation of the manuscript. GB and SH were the principally involved in the design of the BAU. JK undertook the data analysis

This study was undertaken in the Emergency Department, Royal Melbourne Hospital, Grattan St Parkville, 3050.

All correspondence can be addressed to Jonathan Knott in the RMH Emergency Department jonathan.knott@mh.org.au

$+61409420116$

Word Count 2776

This is the author manuscript accepted for publication and has undergone full peer review but has not been through the copyediting, typesetting, pagination and proofreading process, which may lead to differences between this version and the Version of Record. Please cite this article as doi: 10.1111/1742-6723.12905

This article is protected by copyright. All rights reserved. 


\begin{abstract}
Objective

We aimed to assess the impact of a new model of care for patients presenting to the ED with acute behavioural disturbance.
\end{abstract}

\title{
Methods
}

This pre/post intervention study involved creating a dedicated, highly resourced six bed unit, the behavioural assessment unit (BAU). Co-located with the ED at Royal Melbourne Hospital, the unit was designed to fasttrack the admission of patients affected by intoxication, mental illness or psycho-social crisis and provide front-loaded interventions.

\section{Results}

In 12 months from April 1 2016, 2379 patients were admitted to the BAU. They were compared to a similar cohort of 3047 patients from the entire 2015 ED population. The BAU resulted in a decreased wait to be seen (40 minutes [IQR: 17-86] versus 68 minutes [IQR:24-130], $p<0.001$ ), a decreased wait for a mental health review (117 minutes [IQR:49-224] versus 139 minutes [IQR:57-262], $\mathrm{p}=0.001$ ) and a decreased ED length of stay (180 minutes [IQR:101-237] versus 328 minutes [IQR: 227-534], $p<0.001$ ). Patients admitted to the BAU were less likely to have a security code (349 $(14.7 \%)$ versus $538(17.7 \%)$, $p=0.003)$ and less likely to have mechanical restraint (156 episodes $(6.6 \%)$ versus $275(9.0 \%), p<0.001)$ or therapeutic sedation (156 episodes $(6.6 \%)$ versus $250(8.2 \%), p<0.001)$.

\section{Conclusion}

A unit specifically designed to improve the care of patients requiring prolonged ED care due to mental illness and/or intoxication reduces the time spent in the ED and the use of some restrictive interventions. We recommend this model of care to EDs that care for this complex and challenging group of patients.

Key words: Behavioural Emergency, Emergency Psychiatry, Patient Flow, Restrictive Interventions

This article is protected by copyright. All rights reserved. 


\section{Introduction}

Acute behavioural disturbance is a medical emergency. It is an increasingly common clinical problem facing health services and Emergency departments (EDs), ${ }^{1}$ and poses a significant direct risk to patient safety as well as to the welfare of staff, the public, and hospital property. ${ }^{2}$ Patients with acute behavioural disturbance are not a homogenous cohort. Previous studies into the causes of acute behavioural disturbance have demonstrated a relationship to drug and alcohol misuse, drug induced psychosis, exacerbation of a preexisting mental health diagnosis, or an underlying organic illness. ${ }^{3,4}$ A primary mental health illness (including psychosis) accounts for only $15 \%{ }^{5}$

In the acute setting, the cause of an acute behavioural disturbance may be hard to differentiate and the initial management of this patient group requires the use of de-escalation strategies, an appropriate environment, highly trained staff and adequate clinical resources to protect the safety and dignity of all concerned. ${ }^{6}$

Care of acute behavioural disturbance in the ED is particularly challenging, often requiring more resources and specialized care than other patient groups. ${ }^{7}$ A study of the mental health population within EDs, a substantive proportion of those patients with acute behavioural disturbance, shows that this patient group have been observed to have a disproportionately extended ED length of stay with significant variation in their management. $^{8}$

In Australia, there have been calls to improve the management of this population, including within the ED. ${ }^{9}$ Barriers to providing optimal care to this patient group include environmental factors such as limited appropriate physical space, concern for safety and security, and access to mental health services; as well as patient factors, such as aggression, intoxication and difficulties differentiating between mental health issues and social disorganisation. ${ }^{10}$ There is a clear need to address these barriers and revise the care provided to this vulnerable population.

In mid-2015, following the Travis report, ${ }^{11}$ Melbourne Health received funding from the Victorian Department of Health to establish a behavioural assessment unit (BAU), comprising six behavioural assessment beds. The newly established area is managed by, and co-located with, the ED. The BAU model of care aims to create a safe and therapeutic environment for behaviourally disturbed patients of any aetiology including; 
patients with acute and chronic substance abuse; patients in psychosocial crisis; patients with acute psychiatric conditions and patients recovering from acute toxidromes secondary to a drug overdose not at risk of requiring intubation.

The philosophy of the unit is to fast-track the assessment and management of behaviourally disturbed patients presenting to the ED in an environment that has been specifically designed to be safe and secure, allow close observation, provide timely access to specialist expertise, and facilities for the appropriate use of sedation and restraint when required, irrespective of the patient's primary diagnosis. At the inception of the project we believed that the need for early intervention was common across each patient cohort and, once a therapeutic environment was created, directed management of the underlying clinical problem could then be safely addressed.

This study aims to assess the impact of the introduction of the BAU on patient outcomes, process and quality of care. 


\section{Methods}

\section{Site and Setting}

Royal Melbourne Hospital is a tertiary centre on the edge of the central business district. There are 72 000 ED presentations per annum with approximately $45 \%$ being admitted. The ED has access to Emergency Mental Health clinicians (EMH) 24 hours a day, a dedicated drug and alcohol clinician three days a week and an on-call toxicology specialist 24 hours a day. There is a 20-bed short stay observation unit designed for patients who will remain under the exclusive care of Emergency Medicine for up to 24 hours.

This study is an evaluation of the impact on patients with an acute behavioural disturbance following the commissioning of the BAU. The BAU is a discrete six bed unit designed to improve privacy and decrease stimulation. It provides specialised care to all patients with acute behavioural disturbance. Specifically, behaviour influenced by drugs and alcohol, drug intoxication, mental illness and social crisis. In the majority of cases, a combination of these acute or chronic triggers will be present. The unit is not a Psychiatric Emergency Care Centre (PECC) as described under the Australasian Health Facilities Guidelines of the Australasian Health Infrastructure Alliance. ${ }^{12}$ PECCs are specifically designed for people presenting to the ED with acute mental health illness who may have a length of stay of to 72 hours. They are closed wards devoid of ligature points and have lockable single bedrooms. Our unit is open; it has four cubicles and two single rooms that are not locked. The two cubicles nearest to, and opposite, the nurses' station are used for patients who have taken overdoses or need close observation and monitoring following sedation. An interview room with a second point of egress is in the unit. A medication preparation area is immediately adjacent and accessed via swipe card. Both fixed and personal duress alarms systems are installed and the area is monitored remotely by our security staff (located 50 meters away) on closed circuit television. EMH and drug and alcohol clinicians are co-located to improve access to early intervention. There are between two and three nurses in the unit at all times, providing a high nurse/patient ratio. $\mathrm{A}$ psychiatrist and/or psychiatry registrar are located in the unit each morning. The unit was designed to admit patients with an acute behavioural disturbance, with an expected discharge to home within 24 hours. The budget for the unit included a "one off" $\$ 500,000$ payment for capital works to refurbish the 
area (previously used as a rapid assessment ward for general medicine patients) and recurrent costs to provide additional staffing; an additional EMH clinician in the evening, one half-time consultant psychiatrist position and one half-time psychiatric registrar position to cover morning ward rounds. The nursing workforce increased in line with workforce agreements to cover the additional six beds. An additional resident was provided overnight to support the 20 bed short-stay unit, as well as the BAU. As the BAU was gazetted as an inpatient area, cost recovery was budgeted upon the WIES generated by the unit (WIES is the Victorian unit of activity based funding). While not an outcome of this study the first 12 months the revenue generated was able to cover the costs and deliver a small surplus. Admission criteria included a restricted age range of 16 to 65 who are not deemed to be at risk of requiring intubation for airway support. For all admission criteria, clinical discretion is acceptable.

\section{Design}

We conducted a pre/post intervention study based around the establishment of the BAU. The unit was opened progressively from early 2016. The post-BAU cohort in this study was assessed from April 1 2016 when the unit became fully operational; 12 months of data was obtained. The pre-BAU cohort was taken from all 2015 ED presentations.

\section{Outcomes}

The BAU was designed to move patients from the ED to a dedicated, well resourced, low stimulus environment. The primary outcome was length of stay in the ED.

The following secondary outcomes were also assessed:

- Time to ED clinician

- Time to EMH clinician

- Code Grey rates

- Restrictive intervention rates 
A Code Grey is the institution's security alert for an unarmed threat. ${ }^{13}$ Restrictive interventions included the need for physical and mechanical restraint and therapeutic sedation.

\section{Study entrance criteria}

The post-BAU data included all patients admitted to BAU from April 12016 to March 312017. Patients who had a medical diagnosis (renal colic, gastroenteritis etc.) were considered as short-stay unit patients and therefore excluded. The short-stay is a geographically separate 20 bed unit not designed for the BAU population but, at times of access block, short-stay patients may be temporarily admitted to the BAU as boarders.

To establish a comparable pre-BAU population, all ED presentations from 2015 were included if their length of stay was between three and 24 hours, their discharge destination was ultimately home or discharging against medical advice, and the patient age was over 16 years of age. Patients were also included only if their final discharge diagnosis was coded as a mental health issue, psychosocial crisis or related to intoxication.

For each time period, data were extracted from the ED Information system ${ }^{14}$ and the security code grey database, and merged into a single Microsoft Excel worksheet (97-2003 Redmond Illinois).

Sample size and analysis

No a priori sample size was calculated but it was predicted in the BAU business case that approximately 3000 patients with drug related, mental health and other self-harm presentations would be admitted to the BAU per annum. It was expected that this would be of sufficient size to identify clinically important changes to patient care.

Patient characteristics in the two cohorts are presented descriptively. Proportions were compared using chi-square tests, parametric variables were examined with t-test or ANOVA and non-parametric variables using the Mann-Whitney or Kruskal-Wallis test as appropriate.

Data was transferred to Stata for analysis. ${ }^{15}$

Consistent with previous studies, and the large number of variables to be investigated, a significance level of 0.01 was set.

This article is protected by copyright. All rights reserved. 
The study was approved by the Melbourne Health Human Research and Ethics Committee and performed in accordance with the ethical standards laid down in the Declaration of Helsinki.

This article is protected by copyright. All rights reserved. 


\section{Results}

In 2015, there were 67311 ED presentations and the median length of stay was 208 minutes (IQR: 127-330). The median waiting time to see a doctor was 47 minutes (IQR: 18-94). Of these, 3047 were selected as the pre-BAU cohort. Between April 12016 and March 312017 there were 72839 ED presentations and the median length of stay was 203 minutes (IQR: 123-314). The median waiting time to see a doctor was 51 minutes (IQR: 20-99). Of these, 3259 were admitted into the BAU. Eight hundred and eighty patients had medical diagnoses and were excluded from the analysis, leaving 2379 patients in the post-BAU cohort.

Table 1 shows the demographics of the two groups. There are no clinically important differences between the two groups.

Figure 1 shows the ED length of stay was decreased in the pre-BAU cohort from 328 minutes (IQR: 227:534) to 180 minutes in the post-BAU cohort (IQR: 101-237), $p<0.001$.

Figure 2 shows the median waiting time to be seen reduced from 68 minutes (IQR: 24-130) to 40 minutes (IQR: 17-86), $p<0.001$,

Similarly, Figure 3 shows the median time to see an EMH clinician reduced once the BAU was operational, from 139 minutes (57-262) to 117 minutes (IQR: 49-224), $p=0.001$.

Table 2 shows the total number of code greys called for each population and the proportion of restrictive interventions. In the pre-BAU cohort, 370 patients had 538 code greys called whilst in the ED. The post-BAU cohort had 259 patients having 349 codes whilst in the ED or in the BAU. There was a small reduction in the rate of patients having mechanical restraint or therapeutic sedation but no overall change in the rate of restrictive interventions.

This article is protected by copyright. All rights reserved. 
The pre-BAU cohort had a mean ED length of stay of 423 minutes with a standard deviation of 265 minutes. The post-BAU cohort had a mean ED length of stay of 210 minutes with a standard deviation of 179 . Setting significance (alpha error) at 0.01 and power (1-beta error) at 0.90 , a post hoc analysis indicates 34 patients would be required in each sample. For the numbers in this study the power to find the effect size seen is 1.0000 . 


\section{Discussion}

The BAU was designed to move a vulnerable population out of the ED into an environment designed to provide less stimulus, better access to the expert care and decrease the need for restrictive interventions.

When compared to an historical control, selected based on ED diagnosis, the total ED length of stay for patients admitted to BAU was significantly less than that observed among the historical controls. We observed fewer code grey events and episodes of mechanical restraint and therapeutic sedation. Although the rate of reduction was small, the opportunity to reduce the restrictive intervention rate is important.

Since the introduction of the National Emergency Access targets (NEAT), Australian hospitals have been progressively improving the throughput of patients, within four hours, closing on the $90 \%$ target. ${ }^{16}$ Certain patient populations have been challenging when it comes to meeting NEAT. These include patients with mental health issues and those who are intoxicated. Mental health, in particular, requires a prolonged initial assessment and corroboration to ensure either safe discharge home or appropriate admission to a psychiatric ward. Typically access to these services is highly constrained. ${ }^{7}$ Intoxicated patients are mostly expected to be discharged when they recover from their toxidrome. The combination of intoxication and mental health crisis is particularly challenging. Our study has demonstrated that a dedicated, purposefully built unit improves the time to be seen, the time to a mental health clinician and substantially decreases the time spent in the ED.

The Department of Health and Human Services in Victoria is seeking to reduce the rate of restrictive interventions, ${ }^{17}$ the Victorian Mental Health Act mandates least restrictive care, ${ }^{18}$ and ED medical and nursing staff seek to provide the best care whilst ensuring patient safety. The BAU resulted in less Code Greys and a reduction in mechanical restraint and therapeutic sedation. Although the size of this impact is small, any reduction is important to the patients involved and the staff that care for them. It is not clear which aspect of the intervention led to this benefit but better access to nursing staff, EMH and drug and alcohol services, together with the low stimulus environment would all be 
expected to be advantageous. Further exploration of this would be better done with a qualitative study.

In our study, only $13 \%$ of patients were identified as having a primary mental health problem (excluding self-harm and hostility). While this figure is consistent with other studies ${ }^{5}$ and supports the supposition that behavioural assessment units are different from PECCs, the proportion is probably greater given that $25 \%$ of diagnoses were coded as "other." Thirty five percent of patients were reported to have a primary toxicology related diagnosis, supporting the need to staff the unit with nurses highly skilled in advanced cardiac life support.

\section{Limitations}

The study was undertaken at a single site with a particular patient population. The results may not be applicable to other sites.

The pre-BAU population was chosen prior to the study commencing. The aim was to identify from the entire 2015 patient population, those patients who would have gone to a BAU if it had been available. However, it is challenging to exactly select the same patient groups. ED discharge coding allows only a single diagnosis and it is possible that patients with intoxication and a minor head injury would have been admitted to BAU in 2015. If coded as a minor injury only, they would not be included in the preBAU cohort. It is possible such a patient would be admitted to short-stay but they may have had their entire stay in the ED. It is unclear how the inclusion or exclusion of small patient sub-groups would affect the results.

It should be noted that Table 1 identifies statistical differences in the two populations but clinically they are very similar. The top four diagnostic categories have a near identical distribution. There is a difference in acuity but it is clinically unlikely to be of importance (e.g. a change in Australasian Triage Scale category 3 of $1.3 \%)$.

As the two populations compared were in different years, it is difficult to determine the impact other ED strategies to improve patient care may have had on the results. Whilst it is not feasible to 
randomise patients to this intervention in a single site, it would be possible to evaluate using a cluster randomised clinical trial across many sites.

It is also possible that the improved outcomes were related to the additional staffing provided for the BAU. As the BAU intervention includes the model of care, the environment and the staff to support the model, the intervention should be considered as a whole. The additional staff within the BAU are a necessary component of the successful intervention.

\section{Conclusion}

A unit specifically designed to improve the care of patients requiring prolonged ED care due to mental illness and/or intoxication reduces the time spent in the ED and the use of some restrictive interventions. As a consequence of the type of patient admitted to the BAU we recommend that they are managed by advanced cardiac life support trained nurses and have access to a multidisciplinary team that can address their mental health issues, substance use issues and significant psychosocial needs. We recommend this model of care to EDs that care for this complex and challenging group of patients.

\section{Acknowledgments}

Laurie Showler assisted with the literature review. 
Table 1: Comparison of the pre-BAU and Post-BAU populations

\begin{tabular}{|c|c|c|c|}
\hline Variable & $\begin{array}{l}\text { Pre-BAU } \\
(n=3047)\end{array}$ & $\begin{array}{l}\text { Post-BAU } \\
(n=2379)\end{array}$ & \\
\hline Age (median, IQR) & $34(25-46)$ & $33(24-46)$ & $p=0.042$ \\
\hline Male $(n, \%)$ & $1729(56.7)$ & $1282(53.9)$ & $p=0.036$ \\
\hline $\operatorname{ATS}^{\dagger}(\mathrm{n}, \%)$ & & & $p<0.001$ \\
\hline 1 & $80(2.6)$ & $86(3.6)$ & \\
\hline 2 & $257(8.4)$ & $254(10.7)$ & \\
\hline 3 & $1792(58.8)$ & $1430(60.1)$ & \\
\hline 4 & $837(27.5)$ & $573(24.1)$ & \\
\hline 5 & $81(2.7)$ & $36(1.5)$ & \\
\hline Arrival mode $(n, \%)$ & & & $p<0.001$ \\
\hline Ambulance & $1705(60.0)$ & $1465(61.6)$ & \\
\hline Police & $269(8.8)$ & $144(6.1)$ & \\
\hline Top Diagnoses (n, \%) & & & $P<0.001$ \\
\hline Alcohol related & $497(16.3)$ & $377(15.8)$ & \\
\hline Self-harm & $308(10.1)$ & $273(11.5)$ & \\
\hline Hostility & $256(8.4)$ & $185(7.8)$ & \\
\hline Depression & $225(7.4)$ & $168(7.1)$ & \\
\hline Transient psychosis & $217(7.1)$ & $76(3.2)$ & \\
\hline Anxiety & $198(6.5)$ & $50(2.1)$ & \\
\hline Schizophrenia & $130(4.3)$ & $92(3.9)$ & \\
\hline Stimulant related & $113(3.7)$ & $89(3.7)$ & \\
\hline Other toxicology & $421(13.8)$ & $465(19.5)$ & \\
\hline Other diagnoses & $682(22.4)$ & $604(25.4)$ & \\
\hline
\end{tabular}

${ }^{\dagger}$ ATS $=$ Australasian triage scale, 1 = to be seen immediately, 2 within 10 minutes, 3 within 30 minutes, 4 within 60 minutes and 5 within 120 minutes.

This article is protected by copyright. All rights reserved. 
Table 2: Comparison of Code grey rates and restrictive intervention rates

Variable

Pre-BAU

$(n=3047)$ $370(12.1)$

$538(17.7)$

$388(12.7)$

$339(11.3)$

$275(9.0)$

$250(8.2)$

Restrictive intervention ( $\mathrm{n}, \%)$

Physical restraint $(\mathrm{n}, \%)$
Post-

\section{$\operatorname{BAU}(n=2379)$}

259 (10.9)

$\mathrm{P}=0.159$

$349(14.7)$

$P=0.003$

Any intervention ${ }^{\dagger}(\mathrm{n}, \%)$

Mechanical restraint ( $\mathrm{n}, \%)$

Therapeutic sedation ( $\mathrm{n}, \%)$
$255(10.7)$

$224(9.4)$

$\mathrm{P}=0.025$

$156(6.6)$

$\mathrm{P}=0.043$

$P<0.001$

$156(6.6)$

$\mathrm{P}<0.001$ 
Figure 1: Total length of stay in the Emergency Department (minutes)

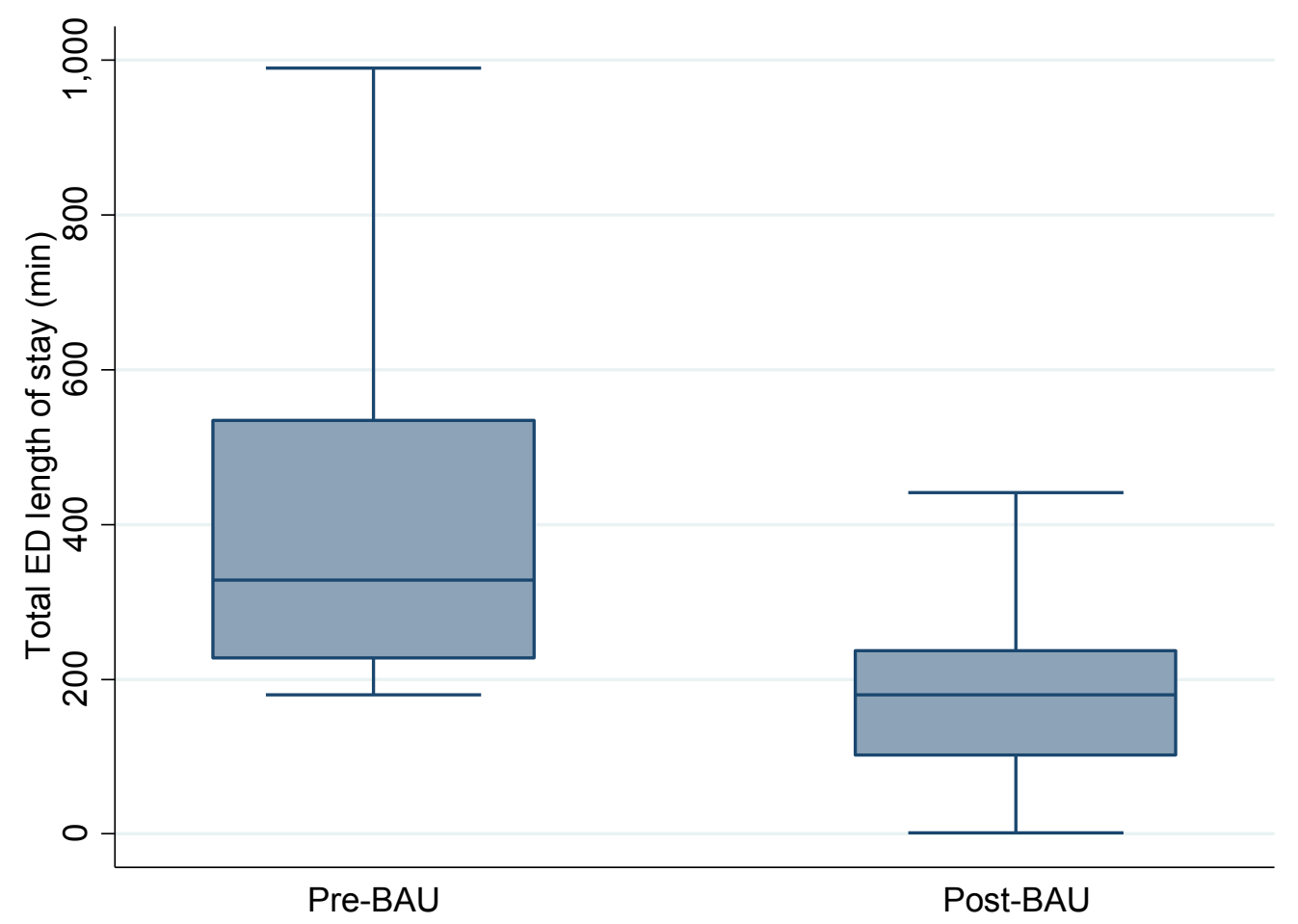

Box shows median and interquartile range, whiskers represent values up to $1.5 \mathrm{x}$ the interquartile range outside the interquartile range. Outside values have been suppressed. 
Figure 2: Waiting time to see a doctor (minutes)

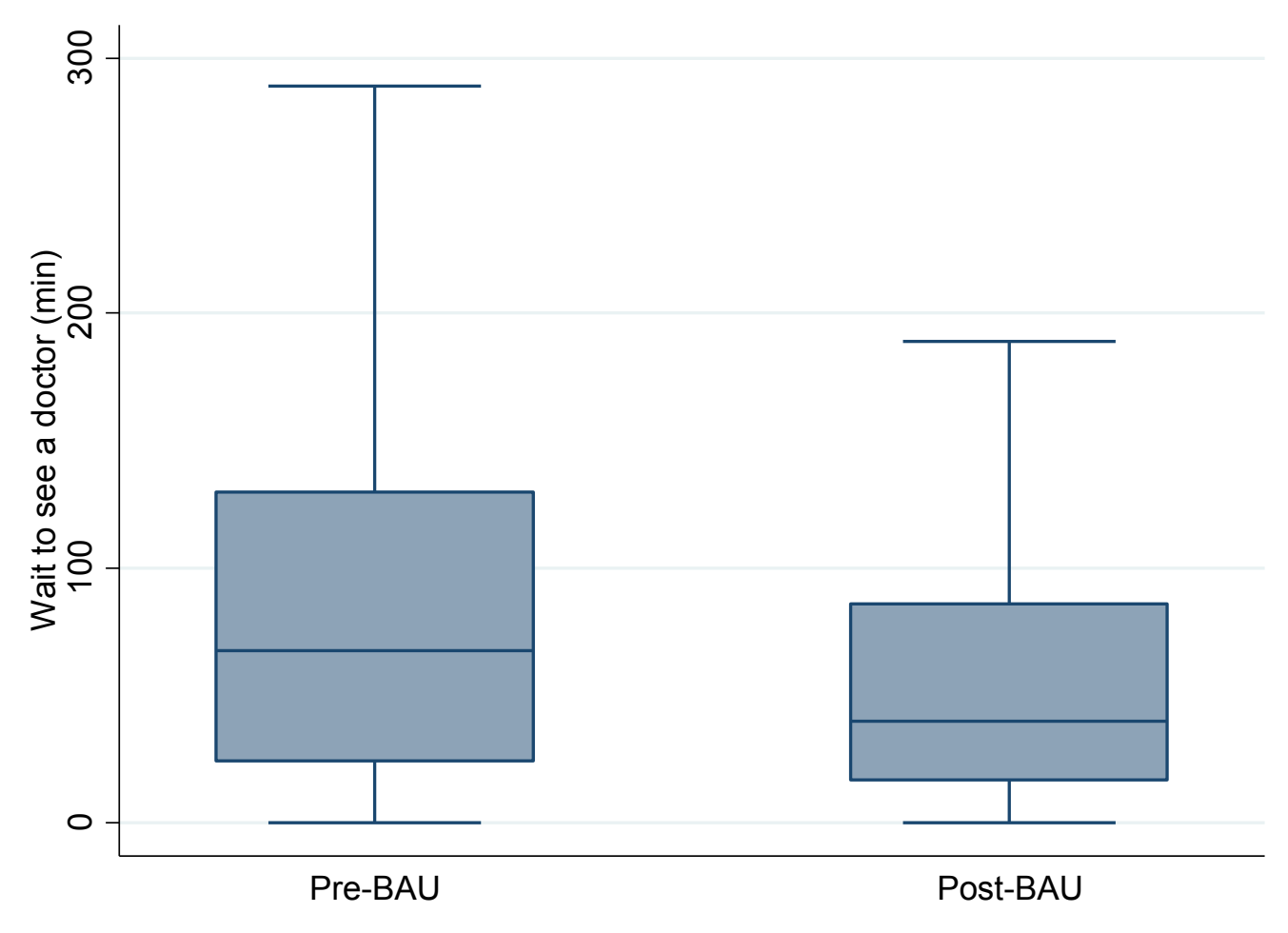

Box shows median and interquartile range, whiskers represent values up to $1.5 \mathrm{x}$ the interquartile range outside the interquartile range. Outside values have been suppressed. 
Figure 3: Time to see a Mental Health clinician in the Emergency Department

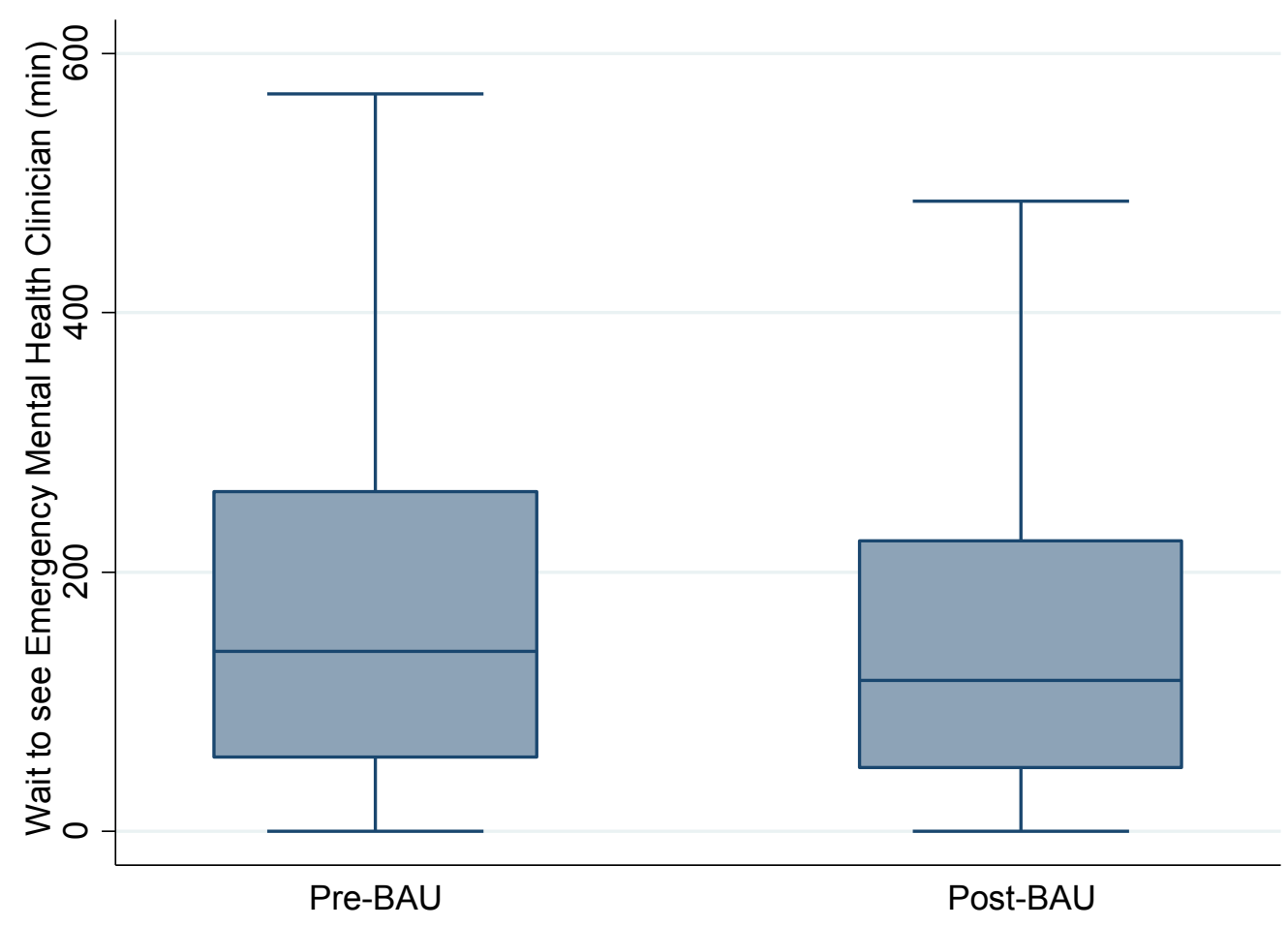

Box shows median and interquartile range, whiskers represent values up to $1.5 \mathrm{x}$ the interquartile range outside the interquartile range. Outside values have been suppressed. 


\section{REFERENCES}

1. Victorian Auditor-General. Occupational Violence against Healthcare Workers. 2015. Avaiable from http://www.audit.vic.gov.au/publications/20150506-Occ-Violence/20150506-Occ-Violence.pdf Last accessed June 152017

2. Calver LA, Stokes B, Isbister GK. Sedation assessment tool to score acute behavioural disturbance in the emergency department. Emerg Med Aust. 2011;23(6):732-40.

3. Brookes JG. The incidence, severity and nature of violent incidents in the emergency department. Emerg Med. 1997;9(1):5-9.

4. Knott JC, Bennett D, Rawet J, Taylor DM. Epidemiology of unarmed threats in the emergency department. Emerg Med Australas. 2005;17(4):351-8.

5. Calver L, Page CB, Downes MA, Chan B, Kinnear F, Wheatley L, et al. The safety and effectiveness of droperidol for sedation of acute behavioral disturbance in the emergency department. Ann Emerg Med. 2015;66(3):230-8. e1.

6. Galletly C, Castle DJ, Dark F, Humberstone V, Jablensky A, Killackey E, et al. Royal Australian and New Zealand College of Psychiatrists clinical practice guidelines for the management of schizophrenia and related disorders. http://journals.sagepub.com/doi/abs/10.1177/00048674166411952016; Available from: http://journals.sagepub.com/doi/abs/10.1177/0004867416641195. Last accessed June 152017

7. Browne V, Knott J, Dakis J, Fielding J, Lyle D, Bruce M, et al. Improving the care of mentally ill patients in a tertiary Emergency Department - Development of a Psychiatric Assessment and Planning Unit PAPU. Aust Psych. 2011;19(4):350 -3.

8. Manchengo PA, Knott JC, Graudins A, Bartley B, Mitra B. Management of mental health patients in Victorian emergency departments: A 10 year follow-up study. Emerg Med Aust. 2015;27(6):529-36.

9. Brakoulias V, Mandali R, Seymour J, Sammut P, Starcevic V. Characteristics of admissions to a recently opened Psychiatric Emergency Care Centre. Aust Psych. 2010;18(4):326-29.

10. Weiland T, Mackinlay C, Hill N, Gerdtz MF, Jelinek GA. Optimal management of mental health patients in Australian emergency departments: Barriers and solutions. Emerg Med Aust. 2011;23(6):677-88.

11. Travis D. Travis review. Vicroian State Government 2014. Available at https://www2.health.vic.gov.au/about/health-strategies/travis-review Last accessed June 152017

12. Alliance AHI. Australasian Health Facility Guidelines. Part B - Health Facility Briefing and Planning Page 30133 - Psychiatric Emergency Care Centre PECC. 2016 [updated 21 December 2016June 14 2017]; Available from: https://aushfg-prod-com-au.s3.amazonaws.com/HPU_B.0133_6_0.pdf. Last accessed June 152017

13. Victorian State Government. Code Grey Standards 2016. Available at https://www2.health.vic.gov.au/health-workforce/worker-health-wellbeing/occupational-violenceaggression/code-grey-black. Last accessed June 152017

14. Symphony. Version 2.29: EMIS group. www.emishealth.com/home/

15. Stata Intercooled. Version 14.2. College Station Tx: StataCorpLP. www.stata.com 
16. Austalian Insitute of Health and Welfare. Emergency Department Care 2014-15. Published by the Australian Governement, 2015. Available at http://www.aihw.gov.au/WorkArea/DownloadAsset.aspx?id=60129553618. Last accessed June 15 2017

17. Providing a safe environment for all. Framework for reducing restrictve interventions. Victorian State Government, 2016. Available at https://www2.health.vic.gov.au/mental-health/practice-and-servicequality/safety/reducing-restrictive-interventions/framework-for-reducing-restrictive-interventions. Last accessed June 152017

18. Mental Health Act 2014, Stat. 14-026 (2014). Victorian Legislation and Parliamentary Documents. Available at

http://www.legislation.vic.gov.au/Domino/Web Notes/LDMS/PubStatbook.nsf/f932b66241ecf1b7ca2 56e92000e23be/0001F48EE2422A10CA257CB4001D32FB/\$FILE/14-026aa\%20authorised.pdf. Last accessed June 152017

This article is protected by copyright. All rights reserved. 


\section{University Library}

\section{- M M I N E R VA A gateway to Melbourne's research publications}

Minerva Access is the Institutional Repository of The University of Melbourne

Author/s:

Braitberg, G;Gerdtz, M;Harding, S;Pincus, S;Thompson, M;Knott, J

Title:

Behavioural assessment unit improves outcomes for patients with complex psychosocial needs

Date:

2018-06-01

Citation:

Braitberg, G., Gerdtz, M., Harding, S., Pincus, S., Thompson, M. \& Knott, J. (2018).

Behavioural assessment unit improves outcomes for patients with complex psychosocial needs. EMERGENCY MEDICINE AUSTRALASIA, 30 (3), pp.353-358. https:// doi.org/10.1111/1742-6723.12905.

Persistent Link:

http://hdl.handle.net/11343/294004 